\title{
Advances in angle-of-arrival and multidimensional signal processing for localization and communications
}

\author{
Laurence Mailaender ${ }^{1 *}$, Sofiene Affes ${ }^{2}$, Markku Juntti $^{3}$ and Marius Pesavento ${ }^{4}$
}

Our ability to determine the direction-of-arrival of a transmitted signal via antenna arrays has improved dramatically in recent years. The invention of the multiple signal classification (MUSIC) and estimation of signal parameters via rotational invariant techniques (ESPRIT) algorithms enabled performance exceeding the resolution limit prescribed by the array dimensions (so-called "super-resolution"). Applications include localization and tracking of airborne, undersea, or ground-based targets, civilian search and rescue operations, emergency caller location via cell towers, precision aircraft navigation and landing, characterization and modeling of spatial/temporal channels, and future location-based services in cellular networks. Angle estimation becomes especially challenging when multipath, interference, array miscalibration, or source correlation is encountered. Most textbook treatments of this topic cover only one-dimensional (1D) angle estimation, implying that the source's movement takes place in the plane of the array. The extension to two-dimensional (2D) AOA (azimuth and elevation) is not only necessary for many realworld applications, but introduces new problems and challenges that do not occur in the 1D case. Furthermore, angular measurements may be combined with other modalities, such as Doppler, delay, delay-difference, polarization, received power, etc., necessitating a multi-dimensional (M-D) approach to direction-finding, location determination, or parametric MIMO channel modeling. Relevant problems involve so-called "pair matching" or "parameter association" between sets of independently obtained arrival angles or other parameters, complexity reduction in approaches requiring M-D search, and non-planar array design. Furthermore, the above techniques and problems can be applied to

\footnotetext{
* Correspondence: Im@lgsinnovations.com

'LGS Innovations, Florham Park, NJ, USA

Full list of author information is available at the end of the article
}

radar, acoustic localization, and nuclear magnetic resonance or acoustic imaging.

We invite the reader to investigate these topics through the articles contained in this Special Issue entitled, "Advances in Angle-of-Arrival and Multidimensional Signal Processing for Localization and Communications."

The article by El Korso, Boyer, Renaux, and Markos entitled, "Statistical Resolution Limit in the Multidimensional Harmonic Retrieval Model: Hypothesis Test and Cramer-Rao Bound Approaches," considers fundamental issues in imaging and localization. It contributes to our understanding of how closely spaced objects can be while still being resolvable as separate, in the case where multiple parameters are estimated.

The article by Mailaender, "Bounds on 2-D Angle-ofArrival Estimation with Separate and Joint Processing," considers fundamental bounds for source localization accuracy, when the two spatial angles are estimated separately, or jointly.

The article by da Costa, Roemer, Haardt, and de Sousa entitled, "Multi-Dimensional Model Order Selection," considers the fundamental problem of deciding how many parameters are actually relevant to the model. Extensions and generalizations of previous results are obtained using tensor techniques.

Liu and Liang's article entitled "Two-Dimensional Frequencies Estimation using Two-Stage Separated Virtual Steering Vector-Based Algorithm" proposes an efficient solution to the pair-matching problem encountered in separate determination of the spatial frequencies across an array.

The article by Barcelo, Vicario, and Seco-Granados, "A Reduced Complexity Approach to IAA Beamforming for Efficient DOA Estimation of Coherent Sources," develops iterative algorithms for 2D angle estimation in environments with coherent sources with low complexity. 
Kowakami and Ohtsuki pick up a similar theme in "Localization Using Iterative Angle-of-Arrival Methods Sharing Snapshots of Coherent Subarrays," which presents a two-stage coarse/fine solution to the problem.

The article by Racine and Grenier, "Improvement on EVESPA for Beamforming and Direction of Arrival Estimation," also specifically deals with the problem of coherent sources, with improvements from making use of the signal subspace.

Bousnina, Stephenne, Affes, and Samet contribute an article entitled, "A New Low-Complexity Angular Spread Estimator in the Presence of Line-of-Sight with Angular Distribution Selection," incorporating angle spread estimation into the direction of arrival estimation problem in nonlinear sensor arrays.

The article by Moghaddam and Akbari entitled, "A Novel ULA-based Geometry for Improving AOA Estimation," proposes off-axis antenna elements and demonstrates low-complexity angle estimation.

In "TSaT-MUSIC: A Novel Algorithm for Accurate and Rapid Ultrasonic 3D Localization," the authors Mizutani, Ito, Sugimoto, and Hashizume propose a method for indoor localization using ultrasonic acoustic signals.

Finally, Callmer, Tornqvist, Gustafsson, Svensson, and Carlbom give us "Radar SLAM using Visual Features," where a ship is able to estimate its own position using radar returns.

The Guest Editors wish to thank all the contributing authors and especially the anonymous reviewers whose efforts help us maintain the high standards of the EURASIP journal. We also thank our Editor, Prof. Phillip Regalia, and the entire Publication Staff at Hindawi and BioMed for their dedication and hard work.

Author details

${ }^{1}$ LGS Innovations, Florham Park, NJ, USA ${ }^{2}$ INRS-EMT, Montreal, QC, Canada ${ }^{3}$ Centre for Wireless Communications, University of Oulu, Oulu, Finland

${ }^{4}$ Darmstadt University of Technology, Darmstadt, Germany

Received: 28 August 2011 Accepted: 27 October 2011

Published: 27 October 2011

doi:10.1186/1687-6180-2011-94

Cite this article as: Mailaender et al:: Advances in angle-of-arrival and multidimensional signal processing for localization and

communications. EURASIP Journal on Advances in Signal Processing 2011 2011:94.

\section{Submit your manuscript to a SpringerOpen ${ }^{\mathcal{O}}$ journal and benefit from:}

- Convenient online submission

- Rigorous peer review

- Immediate publication on acceptance

- Open access: articles freely available online

- High visibility within the field

- Retaining the copyright to your article

Submit your next manuscript at $>$ springeropen.com 